\title{
A PSEUDOCOMPACT TYCHONOFF SPACE THAT IS NOT STAR LINDELÖF
}

\author{
YANKUI SONG
}

(Received 2 March 2011)

\begin{abstract}
Let $P$ be a topological property. A space $X$ is said to be star $P$ if whenever $\mathcal{U}$ is an open cover of $X$, there exists a subspace $A \subseteq X$ with property $P$ such that $X=\operatorname{St}(A, \mathcal{U})$, where $\operatorname{St}(A, \mathcal{U})=\bigcup\{U \in$ $\mathcal{U}: U \cap A \neq \emptyset\}$. In this paper we construct an example of a pseudocompact Tychonoff space that is not star Lindelöf, which gives a negative answer to Alas et al. ['Countability and star covering properties', Topology Appl. 158 (2011), 620-626, Question 3].
\end{abstract}

2010 Mathematics subject classification: primary 54A25; secondary 54D20.

Keywords and phrases: pseudocompact, star countable, star $\sigma$-compact, star Lindelöf.

\section{Introduction}

By a 'space' we mean a topological space. Let $X$ be a space and $\mathcal{U}$ a collection of subsets of $X$. For $A \subseteq X$, let $\operatorname{St}(A, \mathcal{U})=\bigcup\{U \in \mathcal{U}: U \cap A \neq \emptyset\}$.

Definition 1.1 $[2,9]$. Let $P$ be a topological property. A space $X$ is said to be star $P$ if whenever $\mathcal{U}$ is an open cover of $X$, there exists a subspace $A \subseteq X$ with property $P$ such that $X=\operatorname{St}(A, \mathcal{U})$. The set $A$ will be called a star kernel of the cover $\mathcal{U}$.

The term star $P$ was coined in [9] and used in [1, 2], but certain star properties, specifically those corresponding to ' $P=$ finite' and ' $P=$ countable', were first studied by van Douwen et al. in [8] and later by many other authors. A survey of star covering properties with a comprehensive bibliography can be found in [4]. Here, we use the terminology from $[2,4]$. In $[8,9]$ a star finite space is called starcompact and strongly 1-starcompact, and a star countable space is called star Lindelöf and strongly 1-star Lindelöf. In [7], a star $\sigma$-compact space is called $\sigma$-starcompact. From the definitions, we have the following diagram:

$$
\text { star countable } \longrightarrow \text { star } \sigma \text {-compact } \longrightarrow \text { star Lindelöf }
$$

In [2], Alas et al. studied the relationships of star $P$ properties for $P \in$ \{Lindelöf, $\sigma$ compact, countable\} with other Lindelöf type properties and asked the following question.

(C) 2011 Australian Mathematical Publishing Association Inc. 0004-9727/2011 \$16.00 
QUESTION 1.2 [2, Question 3]. Is a pseudocompact Tychonoff space star Lindelöf?

The purpose of this note is to construct an example which gives a negative answer to this question.

Let $\mathfrak{c}$ denote the cardinality of the set of all real numbers. As usual, a cardinal is an initial ordinal and an ordinal is the set of smaller ordinals. Every cardinal is often viewed as a space with the usual order topology. Other terms and symbols that we do not define follow [3].

\section{Main results}

In this section we construct an example of a pseudocompact Tychonoff space that is not star Lindelöf. For a Tychonoff space $X$, let $\beta(X)$ denote the Čech-Stone compactification of $X$.

THEOREM 2.1. There exists a pseudocompact Tychonoff space which is not star Lindelöf.

PROOF. Let $D=\left\{d_{\alpha}: \alpha<\mathfrak{c}\right\}$ be a discrete space of cardinality $\mathfrak{c}$ and let

$$
X=(\beta(D) \times(\mathfrak{c}+1)) \backslash((\beta(D) \backslash D) \times\{\mathfrak{c}\})
$$

be the subspace of $\beta(D) \times(\mathfrak{c}+1)$. As was shown by Noble [5], $X$ is pseudocompact; in fact, it has a countably compact, dense subspace $\beta(D) \times \mathfrak{c}$.

Next, we show that $X$ is not star Lindelöf. For each $\alpha<\mathfrak{c}$, let

$$
U_{\alpha}=\left\{d_{\alpha}\right\} \times[0, \mathfrak{c}] .
$$

Let us consider the open cover

$$
\mathcal{U}=\left\{U_{\alpha}: \alpha<\mathfrak{c}\right\} \cup\{\beta(D) \times \mathfrak{c}\}
$$

of $X$. Let $A$ be a Lindelöf subset of $X$ and let

$$
\Lambda=\left\{\alpha:\left\langle d_{\alpha}, \mathfrak{c}\right\rangle \in A\right\} .
$$

Then $\Lambda$ is countable, since $\left\{\left\langle d_{\alpha}, \mathfrak{c}\right\rangle: \alpha<\mathfrak{c}\right\}$ is a discrete closed subset of $X$.

Let

$$
A^{\prime}=A \backslash \bigcup\left\{U_{\alpha}: \alpha \in \Lambda\right\} .
$$

If $A^{\prime}=\emptyset$, then there exists an $\alpha_{0}<\mathfrak{c}$ such that $A \cap U_{\alpha_{0}}=\emptyset$, hence $\left\langle d_{\alpha_{0}}, \mathfrak{c}\right\rangle \notin$ $\operatorname{St}(A, \mathcal{U})$, since $U_{\alpha_{0}}$ is the only element of $\mathcal{U}$ containing the point $\left\langle d_{\alpha_{0}}, \mathfrak{c}\right\rangle$. On the other hand, if $A^{\prime} \neq \emptyset$, then $A^{\prime}$ is closed in $A$ and $A^{\prime}$ is Lindelöf and $A^{\prime} \subseteq \beta(D) \times \mathfrak{c}$, hence $\pi\left(A^{\prime}\right)$ is a Lindelöf subset of a countably compact space $\mathfrak{c}$, where $\pi: \beta(D) \times \mathfrak{c} \rightarrow \mathfrak{c}$ is the projection. Hence, there exists $\alpha_{1}<\mathfrak{c}$ such that $\pi\left(A^{\prime}\right) \cap\left(\alpha_{1}, \mathfrak{c}\right)=\emptyset$. Choose $\alpha<\mathfrak{c}$ such that $\alpha>\alpha_{1}$ and $\alpha \notin \Lambda$. Then $\left\langle d_{\alpha}, \mathfrak{c}\right\rangle \notin \operatorname{St}(A, \mathcal{U})$, since $U_{\alpha}$ is the only element of $\mathcal{U}$ containing $\left\langle d_{\alpha}, \mathfrak{c}\right\rangle$ and $U_{\alpha} \cap A=\emptyset$, which shows that $X$ is not star Lindelöf, which completes the proof. 
REMARK 2.2. Alas et al. [1] show there is an example of such a space by using the example due to Shakhmatov [6]. Shakhmatov's example is very complicated, but it has a point-countable base. The construction of Theorem 2.1 is simpler than theirs.

\section{Acknowledgement}

The author would like to thank Mr Shuicong Quan for his kind help and valuable suggestions.

\section{References}

[1] O. T. Alas, L. R. Junqueira, J. van Mill, V. V. Tkachuk and R. G. Wilson, 'On the extent of star countable spaces', Cent. Eur. J. Math. 9(3) (2011), 603-615.

[2] O. T. Alas, L. R. Junqueira and R. G. Wilson, 'Countability and star covering properties', Topology Appl. 158 (2011), 620-626.

[3] R. Engelking, General Topology, 2nd edn, Sigma Series in Mathematics, 6 (Heldermann, Berlin, 1989).

[4] M. V. Matveev, 'A survey on star-covering properties', Topological Atlas, Preprint No. 330, 1998.

[5] N. Noble, 'Countably compact and pseudocompact products', Czechoslovak Math. J. 279(4) (1985), $825-829$.

[6] D. B. Shahmatov, 'On pseudocompact spaces with point base', Dokl. Akad. Nauk SSSR 19 (1969), 390-397.

[7] Y. Song, 'On $\sigma$-starcompact spaces', Appl. Gen. Topol. 9(2) (2008), 293-299.

[8] E. K. van Douwen, G. K. Reed, A. W. Roscoe and I. J. Tree, 'Star covering properties', Topology Appl. 39 (1991), 71-103.

[9] J. van Mill, V. V. Tkachuk and R. G. Wilson, 'Classes defined by stars and neighbourhood assignments', Topology Appl. 154 (2007), 2127-2134.

YANKUI SONG, Institute of Mathematics, School of Mathematical Science, Nanjing Normal University, Nanjing 210046, PR China

e-mail: songyankui@njnu.edu.cn 